\title{
Transient Gas Phase Interaction Effects During Droplet-Stream Combustion
}

Department of Mechanical Engineering - COPPE

Federal University of Rio de Janeiro - UFRJ

P.O. Box 68503

21945-970 Rio de Janeiro, RJ, Brazil

\author{
Roger H. Rangel \\ rhrangel@uci.edu \\ Department of Mechanical and \\ Aerospace Engineering \\ University of California, Irvine \\ 92697-3975 Irvine, CA, USA
}

Gas-phase transient effects during the combustion of an infinite linear array of droplets are studied in the limit of infinite Damköler number and in the absence of surrounding convective effects. The present study stems from the quantification of truncation-distance effects on the droplet mass vaporization rate, flame position and flame shape in quasisteady numerical simulations. The solution domain is initially split into near and far-field subdomains. Within each subdomain, analytical grid generation techniques are applied allowing the development of appropriate finite-difference expressions, the control of grid point distribution and the treatment of outflow boundary conditions. The interdroplet distance effect is studied and results for the droplet mass vaporization rate and flame behavior are presented. The results show the existence of different flame regimes ranging from isolated to merged flame conditions and similar vaporization rate evolution for different interdroplet distances.

Keywords: droplet vaporization, droplet combustion, interference effects, transient effects, numerical methods

\section{Introduction}

Efficient energy generation and control of pollutants in a wide range of technological applications are among the important issues motivating the study and understanding of various aspects of liquid spray combustion. In the vast majority of applications, which include diesel and liquid-fueled engines, the physical process of vaporization and combustion is preceded by the breakup of liquid jets in order to increase the condensed-phase surface area, and thus enhance the transport characteristics with the surrounding continuous phase. This atomization process invariably leads to dense sprays, within which interaction effects and deviations from the isolated droplet behavior (Spalding, 1953; Spalding, 1955) become important, and make pure analytical treatments not applicable. Besides, pure quasi-steady results are unable to provide an appropriate description of intrinsically unsteady phenomena which are experimentally observed (Nuruzzaman et al, 1971).

Matched asymptotic expansions were applied for the study of transient effects during the combustion of isolated droplet in quiescent environments (Waldman, 1975). The analysis develops individual quasi-steady and transient solutions for near- and fardroplet fields, respectively. The results indicate an increase of droplet vaporization rates due to gas unsteadiness and flame standoff distances which are consistent with experimental results (Nuruzzaman et al, 1971). Further theoretical studies for isolated droplets applied the $d^{2}$-law profiles as initial conditions in order to isolate pure transient diffusion from fuel vapor accumulation effects (Matalon and Law, 1983; Matalon and Law, 1985). Besides the enhancement in droplet mass vaporization rate, the results also show higher flame temperatures and reduced flame stand-off distances due to transient effects. Quasi-steady initial conditions were also used in ignition studies considering gas-phase transient effects showing the development of the temperature field during the induction period and defining the ignition locus (Chao et al, 1985). The droplet ignition phenomenon was also studied in the presence of acoustic perturbations for nonunity Lewis number (Fachini and Liñán, 1997). Results show that the droplet ignition delay is reduced by the applied acoustic perturbation and possesses a strong dependence on the Damköler number near the lower ignition limit. The influence of gas-phase transient effects during single droplet

Paper accepted March, 2007. Technical Editor: Demetrio Bastos Neto. vaporization was numerically studied for high-pressure situations including sub- and supercritical conditions (Zhu et al, 2001). Results from models considering gas-phase transient and quasi-steady behavior were proposed and compared with experimental data. Analyzing the droplet vaporization rate, results show a linear increase of the unsteady effects with pressure for lower temperatures.

In the case of sprays, analytical treatments are rarely possible due to existing multidimensional effects. Experimental works while capable of providing global information on droplet interaction, generally do not provide detailed characteristics of the physical phenomena. For interaction studies, droplet streams provide simple and reproducible arrangements for numerical and experimental investigations. Recent experimental studies on linear arrays of monodispersed droplets provide velocity, temperature and droplet size datum (Castanet et al, 2005). Droplet temperature results indicate the existence of a period of the droplet lifetime during which the discrete phase temperature remains constant. Besides, experimental data also indicate that the droplet surface regression rate follows de $d^{2}$-law and points to the need of further gas phase studies. In the present work, these results are used in establishing droplet surface boundary conditions.

Numerical and analytical studies of an infinite stream of droplets poses the question on the existence of steady-state solutions for the governing equations. A purely-diffusive cylindrically-symmetric model problem, which allows an analytical solution through the separation of variables method was used to analyze the transient aspects of the solution (Leiroz, 1996). Droplet streams are approximated as liquid cylinders with axially varying surface potentials. The analysis show that, in a cylindrical semi-infinite domain, a nontrivial solution can only be found if the boundary condition specified at a given inner radial position is axially nonuniform. Moreover, for increasing radial distances, the solution tends to the average over the area of the specified boundary condition, which does not correspond to a general droplet stream vaporization or combustion problem. Similarly to the droplet stream, the model problem has homogeneous Neumann boundary conditions on the axial direction. Therefore, despite generating profiles which depend on the axial position, steady-state solutions for infinite droplet streams are not able, in general, to satisfy the ambient conditions imposed by the vaporization or combustion phenomena. The study also shows that truncating the originally semi-infinite solution domain allows quasi-steady solutions to be obtained (Leiroz, 1996). The effect of the domain truncation 
distance on the results are necessary in order to validate the approximation (Leiroz and Rangel, 1995). A small truncation distance effect was observed on a quasi-steady region near the droplet stream, thus allowing interaction effects to be quantified through mass vaporization rate results. However, transient effects are important outside this quasi-steady region where, usually, the reaction zone is present.

In the present work, gas-phase transient effects during dropletstream combustion are numerically studied. The results show the existence of different flame regimes ranging from isolated to merged flame conditions and similar vaporization rate evolution for different interdroplet distances. Differences between transient results and available quasi-steady data (Leiroz, 1997) are explored. Droplet mass vaporization rate results show that gas-phase unsteadiness inhibits interference effects for small times.

\section{Nomenclature}

$a=$ domain truncation distance, $m$

$b=$ half interdroplet distance, $m$

$c_{p}=$ specific heat at constant pressure, $\mathrm{kJ} /(\mathrm{kg} \mathrm{K})$

$D=$ mass diffusion coefficient, $\mathrm{m}^{2} / \mathrm{s}$

$h=$ enthalpy, $\mathrm{kJ} / \mathrm{kg}$

$I=$ heat vaporization to heat of combustion ratio

$J a=J a c o b$ number, $c_{p}\left(T_{\infty}-T_{s a t}\right) / h_{f g}$

$k=$ thermal conductivity, $\mathrm{kJ} /(\mathrm{m} s \mathrm{~K})$

$\dot{m}=$ mass vaporization rate, $\mathrm{kg} / \mathrm{s}$

$r$ = radial coordinate, $m$

$R=$ droplet radius, $m$

$t=$ time, $s$

$T=$ temperature, $K$

$u=$ velocity component, $\mathrm{m} / \mathrm{s}$

$U=$ tangential velocity, $\mathrm{m} / \mathrm{s}$

$V=$ normal velocity, $\mathrm{m} / \mathrm{s}$

$Y=$ mass fraction

\section{Greek Symbols}

$\alpha=$ thermal diffusivity, $\mathrm{m}^{2} / \mathrm{s}$

$\beta=$ coupling function

$\varepsilon \quad=\eta$-transformed coordinate

$\eta=r$-transformed coordinate

$v=$ stoichiometric coefficient

$\zeta=\theta$ or $z$-transformed coordinate

$\theta=$ angular coordinate, $r a d$

$\rho=$ density, $\mathrm{kg} / \mathrm{m}^{3}$

$\Phi=$ velocity potencial, $1 / \mathrm{s}$

\section{Subscripts}

$c$ relative to the physical domain diagonal

$f \quad$ relative to fuel

$f g$ relative to vaporization

flame relative to the flame

$o$ relative to oxidant, outer domain

$r \quad$ relative to radial component

ref relative to reference

$s \quad$ relative to droplet surface

sat relative to saturation

$\theta \quad$ relative to tangential component

$\infty \quad$ relative to undisturbed conditions

\section{Superscripts}

* relative to nondimensional

- relative to average

\section{Analysis}

Under nonconvective ambient conditions, the solution domain can be simplified using symmetry considerations around the droplet stream axis, the droplet equatorial and mean distance planes. The physical domain is depicted in Fig.1 which shows the spherical system of coordinates used and the principal dimensions.

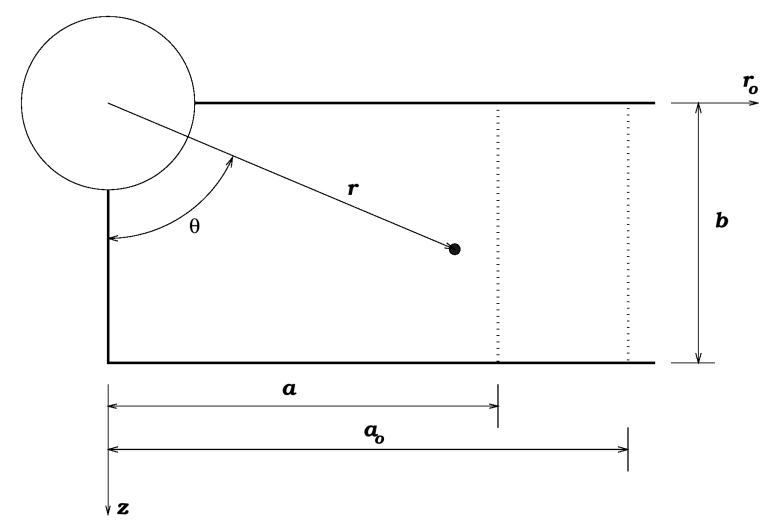

Figure 1. Physical domain and principal dimensions.

In the present study, chemical reaction rates are supposed sufficiently high to guarantee zero oxidant and fuel mass fractions along the droplet surface and on the undisturbed environment, respectively. Assuming unity Lewis number, the nonlinear source terms in the governing equations are eliminated using appropriately defined coupling functions. In terms of the coupling functions defined as

$\beta_{f, o}=\frac{Y_{f}^{*}+Y_{o}^{*} \nu}{Y_{f, s}^{*}+Y_{o, s}^{*} v} ; \beta_{f, t}=\frac{Y_{f}^{*}+J a I T^{*}}{Y_{f, s}^{*}-I} ; \beta_{o, t}=\frac{Y_{o}^{*} \nu+J a I T^{*}}{Y_{o, s}^{*} \nu+I}$

the system of governing equations can be expressed, assuming the flow field as potential and constant physical and thermodynamical properties, as

$$
\begin{aligned}
& \frac{1}{r^{* 2}} \frac{\partial}{\partial r^{*}}\left(r^{* 2} \frac{\partial \Phi^{*}}{\partial r^{*}}\right)+\frac{1}{r^{* 2} \sin \theta} \frac{\partial}{\partial \theta}\left(\sin \theta \frac{\partial \Phi^{*}}{\partial \theta}\right)=0 \\
& \frac{1}{a_{o}^{*}+a^{*}} \frac{\partial \beta_{i}}{\partial t^{*}}+u_{r}^{*} \frac{\partial \beta_{i}}{\partial r^{*}}+\frac{u_{\theta}^{*}}{r^{*}} \frac{\partial \beta_{i}}{\partial \theta}= \\
& =\frac{1}{J a}\left(\frac{1}{r^{* 2}} \frac{\partial}{\partial r^{*}}\left(r^{* 2} \frac{\partial \beta_{i}}{\partial r^{*}}\right)+\frac{1}{r^{* 2} \sin \theta} \frac{\partial}{\partial \theta}\left(\sin \theta \frac{\partial \beta_{i}}{\partial \theta}\right)\right)
\end{aligned}
$$

in $1<r^{*}<b * / \cos \theta, 0<\theta<\pi / 2$ for $t^{*}>0$ and where the velocity components are defined

$$
u_{r}^{*}=\frac{u_{r}}{V_{r e f}}=\frac{\partial \Phi^{*}}{\partial r^{*}}, \quad u_{\theta}^{*}=\frac{u_{\theta}}{V_{r e f}}=\frac{1}{r^{*}} \frac{\partial \Phi^{*}}{\partial \theta}
$$

with boundary conditions

$$
\begin{aligned}
& \frac{\partial \Phi^{*}}{\partial r^{*}}=V_{s}^{*}\left(t^{*}, \theta\right), \quad \beta_{i}=\beta_{i ; s}\left(t^{*}, \theta\right) ; \\
& r^{*}=1, \quad 0<\theta<\pi / 2, \quad t^{*}>0
\end{aligned}
$$




$$
\begin{aligned}
& \Phi^{*} \rightarrow 0, \quad \beta_{i} \rightarrow \beta_{i ; \infty} ; \quad r^{*} \rightarrow \infty, \quad t^{*}>0 \\
& \frac{\partial \Phi^{*}}{\partial \theta}=0, \quad \frac{\partial \beta_{i}}{\partial \theta}=0 ; \quad \theta=0, \quad 1<r^{*}<b^{*}, t^{*}>0 \\
& \frac{\partial \Phi^{*}}{\partial \theta}=0, \quad \frac{\partial \beta_{i}}{\partial \theta}=0 ; \quad \theta=\frac{\pi}{2}, \quad 1<r^{*}<\infty, t^{*}>0 \\
& -\frac{\partial \Phi^{*}}{\partial \theta} \sin \theta+\frac{\partial \Phi^{*}}{\partial r^{*}} b^{*}=0, \\
& -\frac{\partial \beta_{i}}{\partial \theta} \sin \theta+\frac{\partial \beta_{i}}{\partial r} b=0 ; \quad r^{*} \cos \theta=b, 0<\theta<\frac{\pi}{2}, t^{*}>0
\end{aligned}
$$

In defining the boundary conditions, quasi-steady behavior and uniform temperature are assumed for the liquid phase. These assumptions are corroborated by the experimental data (Castanet et al, 2005).

Initial conditions are defined as

$$
\Phi^{*} \rightarrow 0, \quad \beta_{i}=\beta_{i ; \infty} ; 1<r^{*}<b^{*} / \cos \theta, \quad 0<\theta<\pi / 2
$$

which corresponds to the absence of Stefan flow generated by droplet vaporization and unperturbed conditions at infinity prevailing throughout the physical domain.

The coupling conditions at the droplet surface are reduced to

$$
\left.\frac{1}{J a} \frac{\partial \beta_{i}}{\partial r^{*}}\right|_{r^{*}=1}=V_{s}^{*} ; \quad t^{*}>0
$$

where $i$ can be equal to $(f, o) ;(f, t)$ or $(o, t)$ and the values for $\beta_{i ; \infty}$ and $\beta_{i, s}$ are readily obtained from the definitions in Eq.(1) and a Clausius-Clapeyron equilibrium relation. by:

The equations are nondimensionalized using variables defined

$$
r^{*}=\frac{r}{R} ; \quad \Phi^{*}=\frac{\Phi}{V_{r e f} R} ; \quad T^{*}=\frac{T-T_{s a t}}{T_{\infty}-T_{s a t}} ; t^{*}=\frac{V_{r e f} \cdot t}{a+a_{o}}
$$

Where

$$
V_{r e f}=\frac{k}{\rho h_{f g}} \frac{T_{\infty}-T_{s a t}}{R}
$$

The definition of $t^{*}$ in Eq.(12) introduces a nondimensional coefficient for the time derivative term in Eq.(3). Besides, it should be noted that the velocity potential temporal dependence does not reside on Eq.(2), that expresses the mass conservation principle, but on the corresponding boundary condition along the droplet surface Eq.(5).

As in the quasi-steady case, the flame position is determined as the locus where the concentrations of fuel and oxidant vanish. In terms of the $\beta_{f, o}$ coupling function, the flame sheet position is find as

$$
\beta_{f, o}=\frac{1}{Y_{f, s}^{*}} ; \quad t^{*}>0
$$

\section{Numerical Considerations}

The governing equations are solved using the finite-difference method (Mikowycz et al, 1988; Anderson et al, 1984). Initially, the solution domain is split into an inner $\left(1 \leq r^{*} \leq b^{*} / \cos \theta, 0 \leq \theta \leq \theta_{\mathrm{c}}\right.$ and $\left.1 \leq r^{*} \leq a^{*} / \sin \theta, \theta_{\mathrm{c}} \leq \theta \leq \pi / 2\right)$ and an outer $\left(a^{*} \leq r_{o}{ }^{*} \leq \infty, 0 \leq\right.$ $\left.z^{*} \leq b^{*}\right)$ sub-domain. The semi-infinite outer sub-domain is truncated for numerical simulation purposes at $r_{o} *=a_{o} *$ where the outflow boundary conditions are applied. Algebraic transformations are used to regularize the inner sub-domain and to cluster points near the droplet surface in order to control computational requirements (Hoffman, 1992). Within the cylindrical outer subdomain the developed transformations guarantee the matching between inner and outer grids along domain interface, as well as the control of the grid point distribution in the $r_{o}{ }^{*}$ - direction. Complete descriptions on the grid generation procedure within the inner and outer subdomains are presented in Leiroz and Rangel, 1995 and in Leiroz and Rangel, 1997, respectively.

The spatial derivatives in the governing equations are discretized using a second-order accurate scheme throughout the computational domain. The transient term is discretized using a forward first-order scheme. The resulting forward-time centeredspace (FTCS) scheme (Roache, 1972) leads to a system of algebraic equations with the same matrix structure as in the quasi-steady case (Leiroz and Rangel, 1995; Leiroz and Rangel, 1997). Therefore, the iterative procedure used for quasi-steady simulations (Leiroz and Rangel, 1997) remains appropriate for each transient calculation time step.

The system of transformed algebraic equations is solved using the IMSL routine DLSARB (IMSL Library, 1991), which incorporates iterative refinement of the solution (Conte and Boor, 1980) and makes use of the sparseness of the system matrix by means of band storing its coefficients. For each time step, once convergence is achieved, the coordinate transformations are analytically inverted, and the profiles within the physical domain are obtained.

\section{Results}

The numerical scheme was validated by considering limiting cases of isolated droplets under steady-state conditions which allow analytical solutions. Deviations of less than $1 \%$ between numerical and analytical results for the droplet mass vaporization rate and flame stand-off distance were obtained. Besides, these analytical solutions were used to calibrate the clustering parameter $\left(c^{*}\right)$ introduced by the coordinate transformations (Leiroz and Rangel, 1997). Typical value used was $c^{*}=1.01$. Inner sub-domains with an unitary aspect ratio $\left(a^{*}=b^{*}\right)$ were used in the calculations and the smoothness of the obtained solutions presented across the $r_{o}{ }^{*}=a^{*}$ subdomain interface illustrate the appropriateness of the domain splitting procedure. Results were obtained within the time interval required for the flame to completely leave the inner subdomain.

The transient evolution of the temperature field is shown in Fig. 2 for $b^{*}=20$. Since the liquid phase thermal inertia has been neglected in the model, the droplet surface reaches the equilibrium value instantaneously. Therefore, the flame temperature, which is solely dependent on the droplet surface temperature, remains constant and equal to the adiabatic value throughout the simulations. The reaction sheet position and shape are also depicted in Fig 2. For early times $\left(t^{*}=0.12\right.$ and $\left.t^{*}=0.92\right)$, the results show individual spherical flames with increasing stand-off distances, indicating the relative weakness of the droplet interaction effects for early times. Although individual flames are still present for later times $\left(t^{*}=\right.$ 2.12 ), the presence of neighboring droplets distorts the reaction sheet, which assumes an ellipsoidal shape with the major axis 
coinciding with the stream axis. As in the quasi-steady case, the stronger competition for ambient oxidizer near the stream axis is responsible for this preferential deformation direction. This effect is also relevant as the stream-flame regime is reached with the merging of the isolated flames, as can be observed from the reaction sheet deformation near the $z^{*}=b^{*}$ plane for $t^{*}=4.52$. As the merged flame moves away from the droplet stream, a reduction of the flame deformation due to oxidant competition is observed, as a quasicylindrical reaction sheet develops. The obtained flame shapes and regimes are qualitatively consistent with experimental data observed for the combustion of pairs of droplets under microgravity conditions (Mikami et al, 1994). Nevertheless, obtained values for local flame standoff distances are higher than the experimental data due to existing stronger interference effects for droplet-streams.
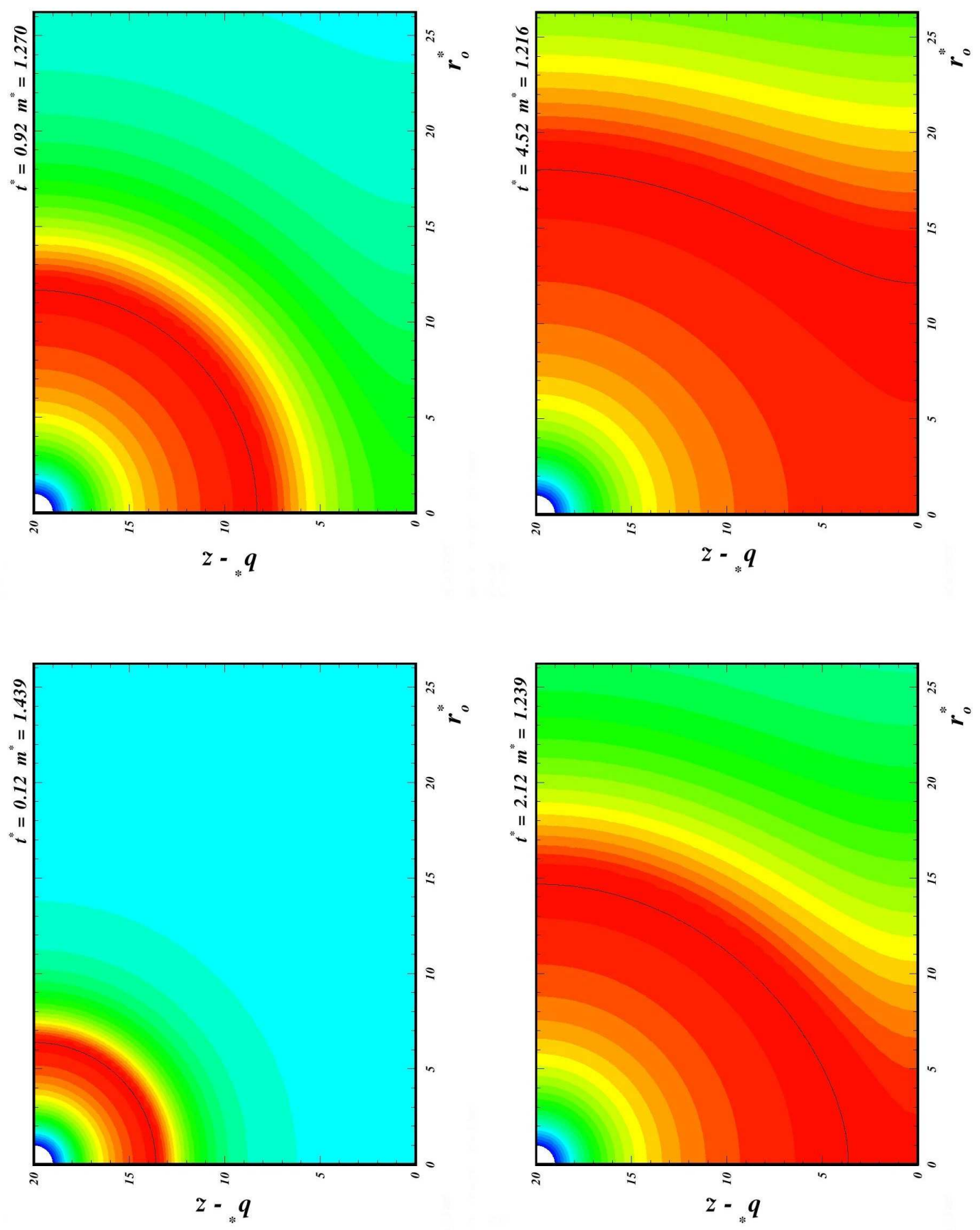

Figure 2. Transient evolution of the temperature field and flame sheet position, $\mathbf{b}^{*}=\mathbf{2 0}$. 
Transient droplet interaction effects on the velocity field are discussed using Fig.3, which shows the velocity potential profile, streamlines and velocity vectors, within the droplet near field for $b^{*}$ $=2$. It is noteworthy that a velocity potential gradient, and thus a nonzero tangential velocity, exists along the droplet surface, which, while in agreement with the potential flow assumption, violates the real-fluid nonslip condition at $r^{*}=1$. The existence of the tangential velocity can be explained by the presence of the neighboring droplets which alter the flow field, introducing a stagnation point along the stream axis at the half-distance symmetry plane $\left(z^{*}=b^{*}\right)$. Therefore, the flow originating from the near-axis droplet region $(\theta$ $\approx 0$ ) is subjected to a higher adverse pressure gradient than the one from near the droplet equatorial plane, leading to an observable surface tangential velocity.

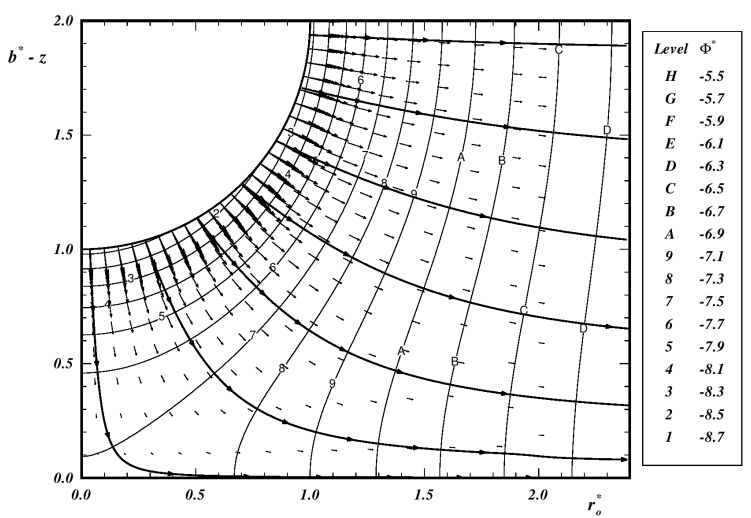

Figure 3. Equipotential lines, streamlines, velocity vectors for a stream of burning droplets - Numerical solution $b^{*}=2$ (Ja $=2-N=129$ points).

It should be mentioned that quasi-steady calculations performed under the same conditions (Leiroz and Rangel, 1997) indicate a negligible velocity potential gradient along the droplet surface, which validated, in that instance, the potential flow assumption. The different transient behavior can be explained with the results presented in Fig. 4, which shows the droplet surface blowing and tangential velocity profiles. The existence of a high gradient region near the droplet stream axis induces a droplet surface blowing velocity profile which decreases towards the droplet equatorial plane. For quasi-steady calculations, the near-axis region of the droplet surface is affected by the low-temperature neighboring droplets thus impairing the vaporization rate and establishing a relation between energy, mass and pressure drop that leads to the negligible tangential velocity.

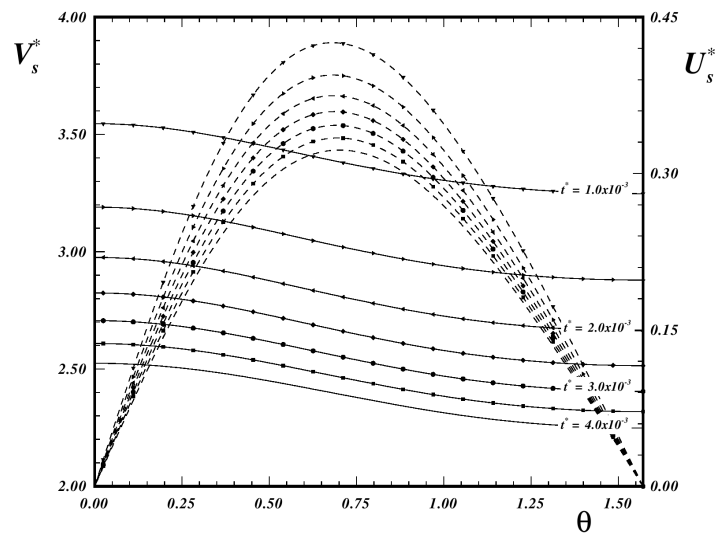

Figure 4. Droplet surface velocity: normal (solid) and tangential (dashed) $b^{\star}=2$.

J. of the Braz. Soc. of Mech. Sci. \& Eng.

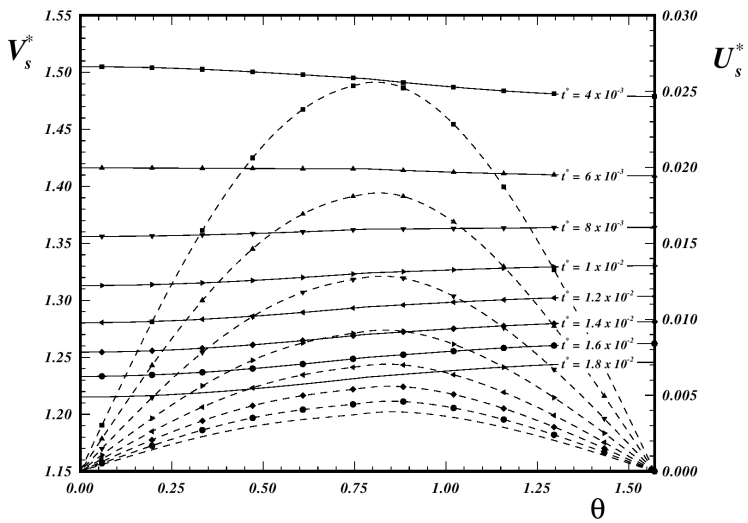

Figure 5. Droplet surface velocity: normal (solid) and tangential (dashed) $b^{\star}=4$.

As the mass fraction and temperature fields evolve, a lowtemperature fuel-rich region develops within the interdroplet spacing and a transition to an increasing blowing velocity profile with the angular position is observed as depicted in Fig. 5 for $b^{*}=$ 4. Results for droplet surface normal velocity shown in Figs. 4 and 5 also indicate higher values for $b^{*}=2$. This positive interference behavior has been experimentally observed during early stages of the combustion of a pair of fuel droplets under microgravity conditions and attributed to thermal radiation effects (Mikami et al, 1994).

Despite the normal velocity behavior shown in Figs. 4 and 5, the tangential velocity remains significant for the studied time intervals, asymptotically tending to a vanishing quasi-steady value. It is worth mentioning that despite the blowing velocity behavior, the droplet surface tangential velocity remains positive for the studied time interval, reaching a maximum in the neighborhood of the inner subdomain diagonal. The droplet surface tangential velocity behavior points to a shear-induced liquid circulation inside the droplet despite the stagnant environmental conditions. In contrast with the single vortex circulation patterns induced by convective flows found in isolated droplets, interaction effects can generate individual vortices in each of the droplet hemispheres, as sketched in Fig.6. The interaction induced vortices would have axes aligned with the droplet stream and thus displace liquid towards the droplet equatorial plane along the droplet surface. Further developments on the droplet-stream combustion model are required in order to quantitatively investigate this phenomenon, which can lead to droplet deformation.

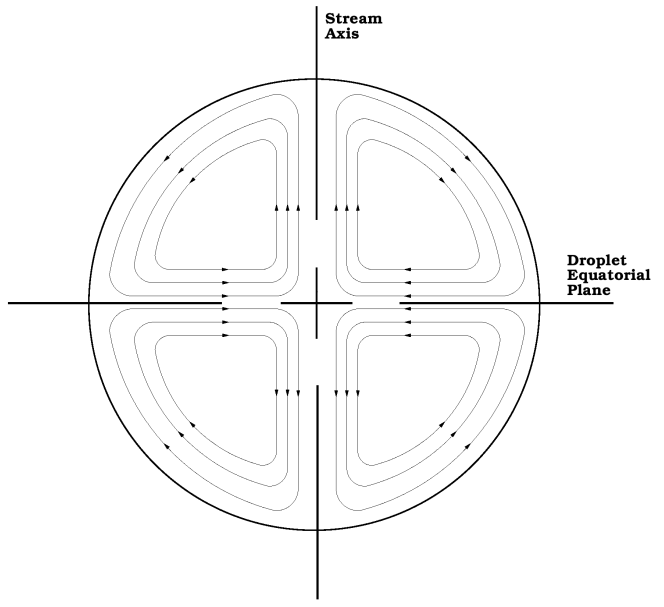

Figure 6. Liquid circulation pattern inside droplets in linear streams. 
The temporal variation of the droplet mass vaporization rate defined as

$$
\dot{m}^{*}\left(t^{*}\right)=\frac{\dot{m}\left(t^{*}\right)}{4 \pi \rho R^{2} V_{r e f}}=\int_{0}^{\pi / 2} V_{s}^{*}\left(\theta, t^{*}\right) \sin (\theta) d \theta, t^{*}>0
$$

is shown in Fig. 7 for different interdroplet spacings. For each time step, converged droplet blowing velocity profiles are used in evaluating the integral in Eq.(15). The subroutines DCSAKM and DCSITG from IMSL (IMSL Library,1991) are used to provide a cubic-spline interpolation of the blowing velocity profile and perform the integration, respectively.

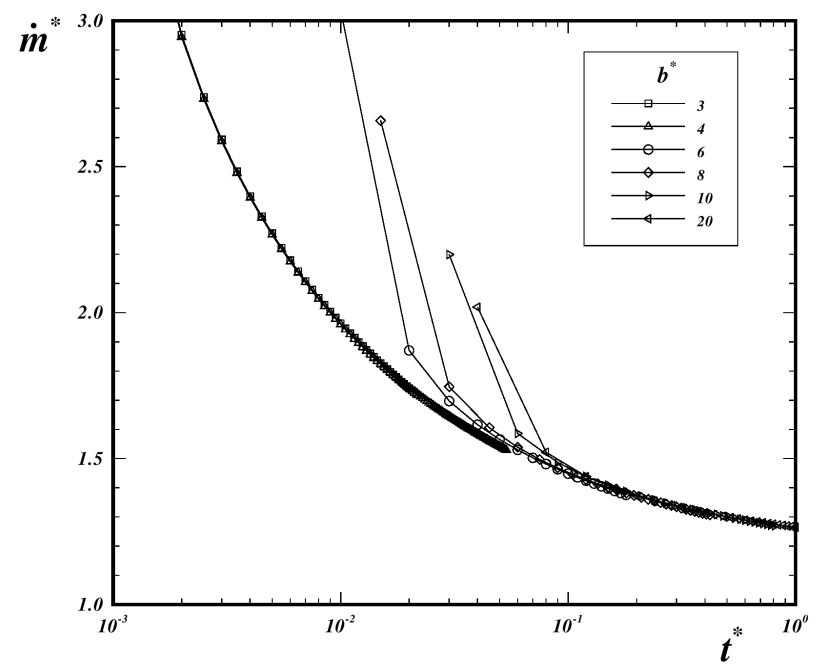

Figure 7. Droplet mass vaporization rate for different interdroplet spacings.

Due to be infinite Damköler assumption, droplet mass vaporization rate results present a discontinuity for initial times as depicted in Fig. 7. Nevertheless, results depicted in Fig. 7 for different spacings converge to a single limiting curve, thus indicating that droplets in streams with different spacings initially vaporize at similar rates. Using a least-square approximation of the numerical data, the correlation for the limiting curve can be written as

$$
\ln \left(\dot{m}^{*}\right)=g_{0} t^{g_{1}}
$$

where $\mathrm{g}_{0}=0.18173$ and $\mathrm{g}_{1}=-0.285078$ with a maximum deviation of less than $1 \%$.

In order to provide further understanding of the similar vaporization rate phenomena, temperature distributions at $t^{*}=0.42$ for interdroplet spacings of 8 and 15 are portrayed in Fig. 8. Flame sheet shape and position are also depicted in Fig. 8. Despite the general discrepancy, temperature distribution in the droplet near field coincide, within the precision of the calculations, for both interdroplet spacings as shown in Fig. 9, thus leading to similar values of droplet mass vaporization rate. Therefore, droplet interaction effects must develop through the gas phase in order to affect the general droplet mass vaporization which is related to the gradient at the droplet surface.
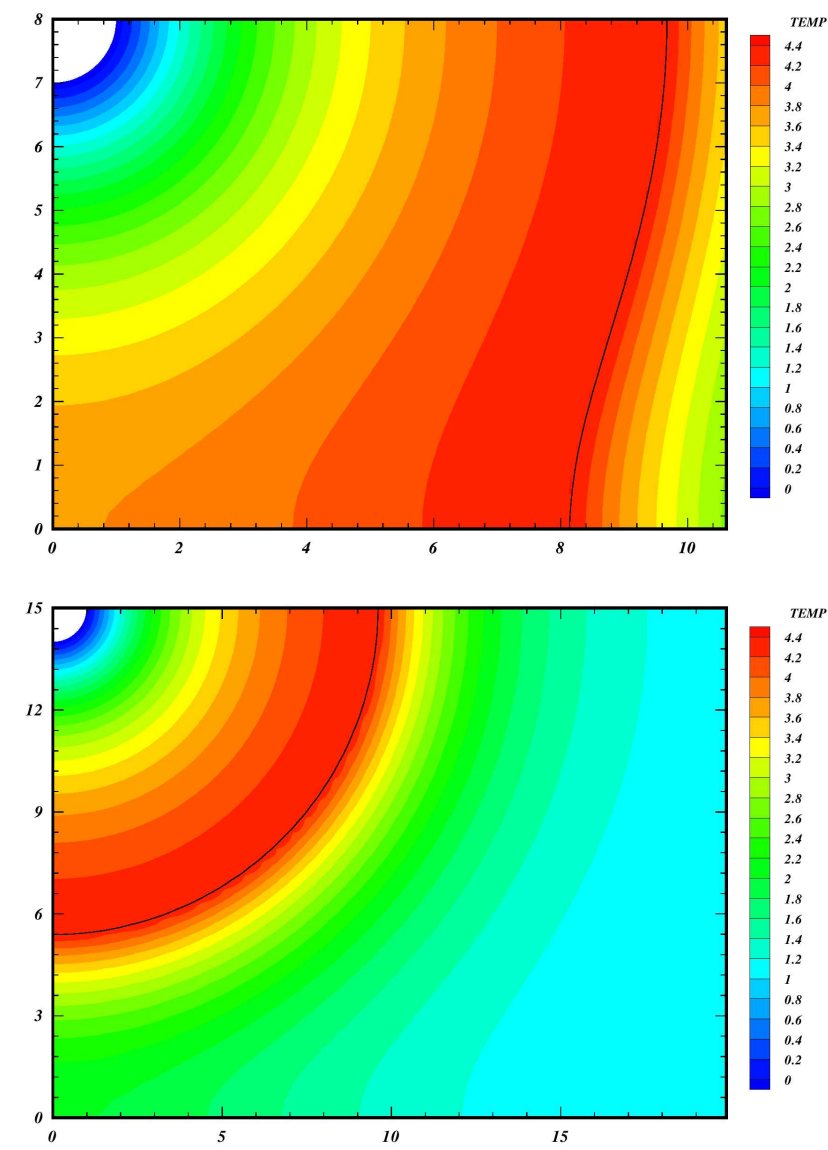

Figure 8. Temperature profiles and flame position for $b^{\star}=8$ (above) and $b^{\star}=15$ (below) $-t^{\star}=0.42$.

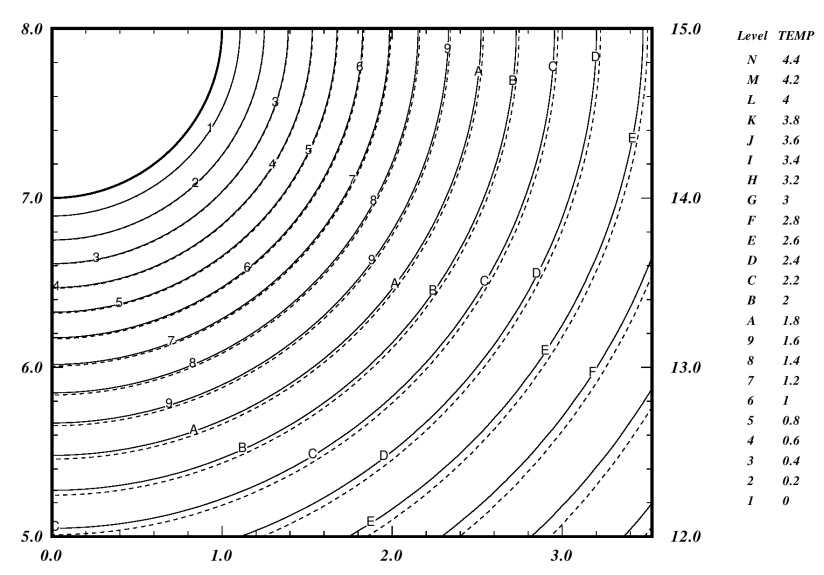

Figure 9. Temperature profiles and flame position for for $b^{\star}=8$ (dashed) and $b^{\star}=15$ (solid) $-t^{\star}=0.42$.

\section{Conclusions}

Gas-phase interaction during droplet-stream combustion in quiescent environments was numerically investigated by extending previously developed quasi-steady analysis. The evolution from isolated to coalesced flame were presented showing the importance of interference effects. Results also allow the analysis of the potential flow assumption showing an observable tangential velocity along the droplet surface. The transient evolution of the droplet surface blowing velocity indicated a non-uniform dependence on the 
angular position over the studied time intervals and positive interference effects. Transient results for the droplet mass vaporization rate and reaction sheet evolution confirm the existence of different time scales within the droplet near and far fields. Within the slow varying near field, results indicated that mass vaporization rates become independent of the interdroplet distance.

\section{Acknowledgment}

The authors acknowledge the financial support from $\mathrm{CNPq}$, Brasília - Brazil (Grant 520315/98-7). This research was also supported in part by the University of California, Irvine and the San Diego Supercomputing Center, through the allocation of computer resources.

\section{References}

Anderson, D., Tannehill, J. and Pletcher, R., 1984, "Computational Fluid Mechanics and Heat Transfer", Hemisphere.

Castanet, G., Lebouché, M. and Lemoine, F., 2005, "Heat and Mass Transfer of Combusting Monodisperse Droplets in Linear Stream", International Journal of Heat and Mass Transfer, Vol. 48, pp. 3261-3275.

Chao, B.H., Matalon, M. and Law, C.K., 1985, "Gas-Phase Transient Diffusion in Droplet Ignition”, Combustion and Flame, Vol. 59, pp. 43-51.

Conte, S. \& Boor, C., 1980, "Elementary Numerical Analysis", McGraw - Hill.

Fachini, F.F. \& A.M. Liñán, 1997, "Transient Effects in Droplet Ignition Phenomenon", Combustion and Flame, Vol. 109, pp. 303-313.

Hoffman, J., 1992, "Numerical Methods for Engineers and Scientists", McGraw Hill.

IMSL, 1991, IMSL Library - Version 2.0, IMSL,Inc.; Park West Tower One, 2500 City West Blvd., Houston, TX, 77042.
Leiroz, A.J.K., 1996, "Numerical Study of Droplet Stream Vaporization and Combustion", Ph.D. Dissertation, University of California, Irvine.

Leiroz, A.J.K. \& Rangel, R.H., 1997, "Flame and Droplet Interaction Effects During Droplet-Stream Combustion at Zero Reynolds Number", Combustion and Flame, Vol. 108, pp. 287-301.

Leiroz, A.J.K., \& Rangel, R.H., 1995, "Numerical Study of DropletStream Vaporization at Zero Reynolds Number", Num. Heat Transfer - Part A: Applications, Vol. 27 pp. 273-296.

Matalon, M. \& Law, C.K., 1983, "Gas-Phase Transient Diffusion in Droplet Vaporization and Combustion", Combustion and Flame, Vol. 50, pp. 219-229.

Matalon, M. \& Law, C.K., 1985, "Gas-Phase Transient Diffusion in Droplet Vaporization and Combustion", Combustion and Flame, Vol. 59, pp. 213-215.

Mikami, M., Kato, H., Sato, J. and Kono, M.,, 1994, "Interactive Combustion of Two Droplets in Microgravity", Proc. 25th International Symposium on Combustion, The Combustion Institute, pp. 431-438.

Minkowycz, W., Sparrow, E., Schneider, G. and Pletcher, R., 1988, "Handbook of Numerical Heat Transfer", John Wiley, New York.

Nuruzzaman, A.S.M., Hedley, A.B. and Beér, J.M., 1971, "Combustion of Monosized Droplet Streams in Stationary Self-Supporting Flames”, Proc. $13^{\text {th }}$ International Symposium of Combustion, pp. 787-799, The Combustion Institute.

Roache, P.J., 1972, "Computational Fluid Dynamics", Hermosa.

Spalding, D.B., 1955, "Some Fundamentals of Combustion”, Chapter 4, Butterworths, London.

Spalding, D.B., 1953, "The Combustion of Liquid Fuels", Proc. 4th International Symposium of Combustion, Cambridge, MA, pp. 847-864, Williams and Wilkins, Baltimore.

Waldman, C.H., 1975, "Theory of Non-Steady State Droplet Combustion", Proc. $15^{\text {th }}$ International Symposium of Combustion, pp. 429442, The Combustion Institute.

Zhu, G.S., Reitz, R.D. and Aggarwal, S.K., 2001, "Gas-Phase Unsteadiness and its Influence on Droplet Vaporization in Sub- and SuperCritical Environments", International Journal of Heat and Mass Transfer, Vol. 44, pp. 3081-3093. 
